\title{
GESTIÓN CREDITICIA Y LA MOROSIDAD DEL BANCO PICHINCHA DEL PERÚ, PERÍODO 2019
}

\author{
CREDIT MANAGEMENT AND DEFAULT OF BANCO PICHINCHA DEL PERÚ, \\ PERIOD 2019
}

Henry Hinostroza Hermoza

Universidad Nacional del Callao

Callao, Perú

ORCID: https://orcid.org/0000-0002-1311-5814

Correo electrónico: hinostrozahh@gmail.com

\section{RESUMEN}

Objetivo: Determinar la incidencia de la gestión crediticia en la morosidad del área de microfinanzas del Banco Pichincha del Perú durante el año 2019, considerando información y data del área de microfinanzas de la agencia en mención. Método: La investigación fue de tipo descriptiva, de enfoque cuantitativo y presenta diseño no experimental. La muestra estuvo conformada por 124 créditos vencidos otorgados por parte del área de microfinanzas del Banco Pichincha del Perú, durante el periodo del 2019. Resultados: Los resultados de la investigación muestran incidencia significativa de la gestión crediticia en la morosidad, encontrando que el 9,12\% de los créditos aprobados por el área de microfinanzas se encuentra con morosidad. Asimismo, en la gestión crediticia, se encuentra una correlación significativa negativa de $-0,113$ en cuanto a evaluación de créditos, y de la misma manera una correlación significativa negativa de $-0,023$ en cuanto a condiciones de crédito. Conclusión: Se concluye que las condiciones del crédito inciden significativamente en la morosidad del área de microfinanzas del Banco Pichincha del Perú, teniendo un grado de correlación significativa negativa de $-0,027$.

Palabras clave: Gestión crediticia; morosidad; evaluación crediticia y condiciones de crédito.

\begin{abstract}
Objective: To determine the incidence of credit management in the delinquency of the microfinance area of Banco Pichincha del Peru during the year 2019, considering information and data from the microfinance area of the agency in question. Method: The research was descriptive, transversal, has a quantitative approach, a non-experimental design, and a quantitative scope. The sample was made up of 124 overdue loans granted by the microfinance area of Banco Pichincha del Peru, during the period of 2019. Results: The investigation's results show a significant incidence of credit management on delinquencies, finding that $9,12 \%$ of the credits approved by the microfinance area are in arrears. Likewise, considering credit management, there is a negative correlation of $-0,113$ in terms of credit evaluation, and in the same way, a negative correlation of $-0,023$ in terms of credit conditions. Conclusion: It is concluded that credit conditions have a significant impact on delinquency in the microfinance area of Banco Pichincha del Peru, showing a negative correlation degree of $-0,027$.
\end{abstract}

Keywords: Credit management; Delinquencies; Credit evaluation and Credit Conditions.

(c) Los autores. Este artículo es publicado por la revista Quipukamayoc de la Facultad de Ciencias Contables, Universidad Nacional Mayor de San Marcos. Este es un artículo de acceso abierto, distribuido bajo los términos de la licencia Creative Commons Atribución 4.0 Internacional (CC BY 4.0) [https://creativecommons.org/licenses by/4.0/deed.es] que permite el uso, distribución y reproducción en cualquier medio, siempre que la obra original sea debidamente citada de su fuente original. 


\section{INTRODUCCIÓN}

En el Perú, existe un mercado desarrollado con una demanda creciente de préstamos dentro de la categoría microfinanzas debido a la importancia de las microempresas para la actividad económica del país y de Lima en particular. Esta importancia radica entre otras cosas, en el alto porcentaje que representan éstas, del total de empresas existentes. Según el Instituto Nacional de Estadística e Informática, de 1 millón 106 mil 853 empresas legalizadas formalmente en Lima Metropolitana, el 93,6\% pertenecen a microempresas, el 5,4\% corresponden a pequeñas empresas y el 0,9\% representaron grandes y medianas empresas. Por lo tanto, Lima se configura como el escenario de mayor relevancia a nivel nacional, en cuanto a producción y generación de empleo (INEI, 2019).

Estos emprendimientos de pequeña y mediana dimensión constituyen la fuerza motora de la actual dinámica económica, constituyendo un porcentaje importante de las unidades económicas creadoras de empleo en la economía nacional.

Esta realidad, vinculada a una serie de políticas económicas propicias, ha creado un entorno favorable en virtud al florecimiento del negocio del sistema microfinanciero. De hecho, el Perú ha sido reconocido como el país con el ambiente más beneficioso para el desarrollo del negocio microfinanciero en todo el mundo; este reconocimiento ha sido otorgado por Economist Intelligence Unit (2016), unidad que no solo situó al país en el ranking del Microscopio Global, sino que además lo ha considerado acreedor del primer lugar del mismo durante seis años consecutivos.

El auge de las microfinanzas impone, sin embargo, un desafío para los oferentes de créditos, pues el incremento del número de clientes es todo un reto en un mercado tan competitivo. De esta forma, es usual que los buenos clientes reciban múltiples opciones de financiamiento por parte de entidades bancarias que compiten entre ellas por colocar sus fondos. En dicha realidad, Ríos-Henckell (2014) menciona que el índice de morosidad del mercado microfinanciero ha venido creciendo; de hecho, la magnitud de la cartera en situación de morosidad, así como la cartera en alto riesgo (que incluye aquellos créditos en morosidad y con refinanciamiento), han aumentado desde su punto más bajo históricamente en 2008 (3,6\% y $4,5 \%$ ), alcanzando para el mes de septiembre de 2013 un $6 \%$ y $7,7 \%$, respectivamente.

Por esta razón, las entidades financieras, que tienen el reto de expandir sus carteras sin comprometer la rentabilidad del negocio, priorizan la búsqueda de una mejora en los mecanismos que minimice la posibilidad de tener clientes que incurran en morosidad.
Con este fin, fue creado en Lima el Banco Pichincha del Perú, el cual inició operaciones en julio de 1964, denominado en aquel momento Financiera y Promotora de la Construcción S.A., adquiriendo en julio de 1986 dicha denominación. El Banco Pichincha se constituye para enfocarse en los requerimientos de la pequeña y mediana empresa, generando para estas una variada gama de productos financieros, desarrollando de este modo operaciones bancarias de consumo, microcrédito y colocación de créditos vía descuento por planilla y casas comerciales, usando también los denominados convenios. Todo esto con el fin de alcanzar el propósito de constituirse como promotor del desarrollo económico en diferentes zonas del Perú.

Dentro de las operaciones en banca minorista, el Banco Pichincha, al igual que otras entidades ya consolidadas, ha incursionado en el mercado microfinanciero y posee un área de microfinanzas y pyme, con el objetivo de apoyar iniciativas empresariales de pequeña escala con el otorgamiento de créditos a tasas competitivas. Sin embargo, aún cuando la cartera de créditos de esta área se ha venido expandiendo, uno de los problemas más relevantes que tiene el Banco Pichincha del Perú es el índice de morosidad en créditos a las microempresas. Una vez desembolsados estos créditos, la agencia, ante la posibilidad de incumplimiento de pago por parte del deudor, efectúa una provisión especifica según el tramo y días de atraso que se encuentre dicho crédito.

En algunos casos, los riesgos de impagos se originan por una evaluación inadecuada del crédito a otorgar, las condiciones en las que se otorga el crédito y la ausencia e interés de la gestión crediticia, que finalmente da lugar a un índice de morosidad muy superior a lo permitido por el Banco Pichincha del Perú.

Por otra parte, desde las políticas de evaluación crediticia aplicadas por la institución, se tiene la poca experiencia de un asesor para evaluar diferentes giros y tipos de negocios. Una considerable propensión al riesgo por parte del área comercial al aceptar riesgos muy elevados a una baja tasa de interés y compras de deuda no realizadas, pueden ser también elementos de peso en la posibilidad de que el cliente incurra en el incumplimiento de sus compromisos.

Para el Banco Pichincha del Perú, particularmente para el área de microfinanzas, existe una amenaza considerable derivada del incremento del índice de morosidad en la cartera del banco. Este aumento viene afectando de manera notable los ingresos de los últimos periodos, reflejados en el estado de resultados del banco; observándose la disminución de las utilidades, el crecimiento de provisiones, los elevados costos operativos y la disminución de 
ingresos netos. Por esta razón, se plantea la necesidad de indagar sobre la influencia de la gestión crediticia en el índice de morosidad de dicho portafolio

En este sentido, durante el 2019, si bien para el Banco Pichincha el índice de morosidad se mantuvo relativamente dentro de los límites establecidos, el área de microfinanzas de dicho banco experimentó un comportamiento ascendente en el índice de morosidad de los créditos del área de microfinanzas, alcanzando incluso el 7,84 \% durante el mes de diciembre.

Aranguiz, Gallegos y Leal (2018) investigaron cómo insertar una metodología para la evaluación de los créditos de los clientes de Fantasía S.A., conllevando a una mejora al momento de la toma de decisiones en la gestión crediticia; permitiendo evaluar la calidad crediticia de cada cliente. Obteniendo como resultado que la validación del modelo se desarrolle con 12 clientes reales de la compañía Fantasía S.A de los casos analizados. La gerencia estuvo de acuerdo con los resultados en 9 de los casos, lo que representa un $81,8 \%$, y en los 3 restantes, que representan el $18,2 \%$, se limitó a realizar observaciones sin rechazar el resultado.

Desde otro punto de vista, Miño (2014) indagó sobre la alta tasa de mora en la cartera de crédito de la Cooperativa de Ahorro y Crédito Pablo Muñoz Vega, y su relación con el modelo de gestión financiero que se utiliza en la misma. En este trabajo el autor se plantea como objetivo desarrollar una propuesta para una orientación dinámica de los procesos del departamento financiero, con la finalidad de ceder créditos a los miembros de la Cooperativa anteriormente mencionada. Concluyó que, el índice de morosidad se relaciona esencialmente con que los protocolos para otorgar los créditos no se ejecutan adecuadamente, lo que acarrea como consecuencia falta de efectividad; sugiriendo diseñar políticas y estrategias para el control y la mejora de dichos procesos.

Martínez, Ríos, y Trejo (2016) analizaron el enfoque a nivel cuantitativo y cualitativo de créditos consolidados, concluyendo que debido a la aplicación de la metodología se presenta una posibilidad de optimización en el modelo actual, a través de la minimización de la creación de provisiones, incrementando la rentabilidad de cada entidad financiera a nivel nacional, y dando cumplimiento a cada supuesto teórico y a cada requerimiento regulatorio, tanto a nivel nacional como internacional en la administración del riesgo crediticio.

Del mismo modo, Turpo (2015) se centró en el análisis del contexto y en las características de cartera activa de la Caja Municipal de Ahorro y Crédito de Arequipa, agencia Macusani, analizando la calidad de la cartera activa, considerando el desenvolvimiento de la tasa de mora, la cartera reestructurada, la cartera vencida, la cartera judicial, la tasa de interés, el número total de los clientes y el número de desembolsos como variables para el periodo 2011-2013. Concluyendo que la calidad de la cartera activa es determinante para las actividades y en la rentabilidad de la Caja Municipal, dado el impacto que tienen los costos asociados a la provisión derivada de la cartera de riesgo sobre el mismo, y las pérdidas asociadas a los créditos que no resultan liquidados por los deudores. Es así que la investigación de Turpo constituye un panorama a considerar en el índice de morosidad de la presente investigación.

De esta forma, como señalan estos trabajos, es claro que la incidencia en el índice de morosidad pueda exacerbarse al no cumplirse con la evaluación de los créditos de manera rigurosa; por otro parte, se demuestra que en algunos casos las deficiencias en dicha evaluación se deben a aspectos inherentes al propio evaluador; su nivel de capacitación, la carga de trabajo que enfrenta e incluso su nivel de experiencia en el área, pueden ser determinantes en la fiabilidad de su criterio.

Otro elemento importante que se desprende del análisis de los trabajos antes mencionados es la incidencia que tiene las propias políticas crediticias de la entidad financiera, teniendo en cuenta que la flexibilidad crediticia puede generar un sobredimensionamiento de las colocaciones, lo cual puede tener un impacto directo en el índice de morosidad.

Aguilar, Camargo, y Morales (2004) señalan que realmente no existe un consenso en cuanto a la discusión sobre cuál es el adecuado indicador "para cuantificar los niveles de morosidad que exhibe la cartera de una organización crediticia" (p. 15). Partiendo de esto, y basándose en la información financiera que publica la Superintendencia de Banca y Seguros, es evidente la utilización habitual en los reportes de al menos tres indicadores de calidad de cartera que permiten cuantificar en valores relativos al nivel de cartera morosa o de mayor riesgo de impago. Estos indicadores son:

Cartera atrasada: Es el ratio que se calcula, teniendo en cuenta las colocaciones vencidas y en cobranza judicial dividido entre el total de colocaciones.

Cartera de alto riesgo: Es el ratio entre colocaciones vencidas, en cobranza judicial, reestructuradas y refinanciadas; dividido entre las colocaciones totales.

Cartera pesada: Es la ratio entre las colocaciones y créditos contingentes clasificados como dudosos, deficientes y pérdidas, divididos entre los créditos directos y contingentes totales. 
De estos tres indicadores mencionados, el más utilizado es el de cartera atrasada, que con frecuencia aparece en la teoría como índice de morosidad, puesto que puede ser obtenido fácilmente a partir de la información contable de las instituciones, información que es, además, de dominio público.

El análisis del comportamiento de dichos indicadores en las entidades microfinancieras, hace pensar que esta variabilidad es originada por las diferencias en políticas de gestión, la tecnología crediticia utilizada y la dinámica interna-externa de sus plazas.

Asimismo, Pérez y Tafur (2015) mencionan que la gestión crediticia se define como la creación de condiciones, construcción de escenarios adecuados, provisión de capacidades e instituciones a los equipos de trabajo; es así como la gestión crediticia se puede considerar como guía para orientar las acciones, previsiones y los objetivos en el tiempo estimado. Así también se gestiona a través del estricto cumplimiento y conocimiento a profundidad de la metodología crediticia, pautas y políticas de riesgo crediticio por parte de los colaboradores que intervienen en el proceso crediticio, cuyos resultados son asegurados por las líneas de supervisión de negocios y riesgos. De la misma manera, el Comité de Basilea, (2009) define la gestión crediticia como aquel proceso que tiene como objetivo la identificación, medición, monitoreo, control y divulgación sobre el riesgo de crédito, incluido en los líneamientos de los objetivos, políticas, procedimientos y acciones establecidas para lograr el propósito establecido.

Lo anterior, sin duda, permite orientar el presente trabajo de investigación en la senda de investigación a seguir, toda vez que facilita elementos importantes para la construcción de las siguientes hipótesis de la investigación: como hipótesis general se encuentra que la gestión crediticia incide significativamente en la morosidad del área de microfinanzas del Banco Pichincha del Perú, y de manera específica, que las condiciones del crédito inciden significativamente en la morosidad del área de microfinanzas del Banco Pichincha del Perú y la evaluación crediticia incide significativamente en la morosidad del área de microfinanzas del Banco Pichincha del Perú.

\section{MATERIALES Y MÉTODOS}

La investigación fue de enfoque cuantitativo, de tipo descriptivo y de diseño no experimental. La muestra fue de tipo censal y estuvo conformada por 124 créditos vencidos otorgados por parte del área de microfinanzas del Banco Pichincha del Perú, durante el periodo 2019. El procesamiento de la estadística se llevó a cabo mediante dos programas, para la estadística descriptiva se utilizó el programa Microsoft Excel y para la estadística inferencial se utilizó el programa estadístico SPSS versión 23.

\section{RESULTADOS}

Se encuentra que el $51,61 \%$ de los clientes con créditos vencidos presentan una mora de 1 a 8 días; el 21,78\% presenta una mora de entre 9 y 15 días; y por último, el $26,61 \%$ presenta entre 16 y 30 días de mora. Asimismo, se encuentra que el $1,61 \%$ son consumo, el 7,26\% de los prestamos vencidos pertenecen a vivienda, el $24,19 \%$ presenta el capital de trabajo, ascendiendo a 30 clientes morosos y el $66,94 \%$ presenta un producto activo fijo, ascendiendo a 83 a clientes.

Se obtiene que los clientes nuevos ascienden a un $42,74 \%$; el $8,87 \%$ de los clientes pertenecen a los clientes en paralelo; el 29,03\% de los clientes pertenecen a un reenganche; y el $19,36 \%$, representado por 24 clientes, pertenecen a clientes de retorno.

De igual manera, se encuentra que el 5,64\% de los clientes se encuentra en un intervalo de 59 a 68 años, el 28,23\% de los clientes, ascendentes a 35 clientes se encuentra en un rango de edad de 21 a 33 años, el 30,65\% de los clientes con crédito vencido se encuentra entre los 47 y 58 años, por último, el 35,48\%, quienes representan el grueso de los clientes se encuentran en un rango de 34 a 46 años.

En la tabla 1, se encuentra que el $45,16 \%$ de los clientes con crédito vencido, recibieron un monto de $S / 1001$ a S/ 5 000, el 20,16\% recibieron un monto de S/ 5001 a S/ 10 000 , el $10,48 \%$ percibieron entre $S / .10,001$ y $S / .20,000$, el mismo porcentaje recibió un monto entre S/ 30001 y S/ 60 000, el 4,84\% recibieron un monto entre S/ 20001 y S/ 30 000 , el mismo porcentaje recibió hasta $\mathrm{S} / 1000$, el 3,23\% percibió un monto de S/ 60001 a S/ 90000 soles y el 0,81\% recibieron entre S/ 90001 y S/ 120000 soles.

En la tabla 2, se encuentra que al 54,84\% de los clientes con crédito vencido les aprobaron el crédito en un intervalo de 7 a 12 meses; el 19,35\% obtuvo un plazo de entre 13 a 18 meses; el 11,29\% de los clientes aplicaron a un plazo de entre 1 a 6 meses; el 7,26\% obtuvo créditos entre 19 y 24 meses; el 4,03\% obtuvo un crédito en un plazo de entre 31 y 36 meses; el 2,42\% aplicó a un rango de plazo de entre 25 y 30 meses; por último, el $0,81 \%$ obtuvo un crédito entre 43 y 48 meses. Se observa que no existe clientes con crédito vencido que tengan un plazo entre 37 y 42 meses.

Finalmente, el $87,10 \%$ de los clientes con créditos vencidos no cuentan con un aval; el 4,03\% de los clientes, ascendentes a 5 clientes, cuentan con un aval con ingreso y el $8,87 \%$ de clientes cuentan con un aval sin ingreso. 
Tabla 1

Monto desembolsado

\begin{tabular}{lccc}
\hline Rango de monto desembolsado & Frecuencia & Porcentaje & Porcentaje Acumulado \\
\hline Hasta S/ 1 000 & 6 & $4,84 \%$ & $4,84 \%$ \\
S/ 1 001- S/ 5000 & 56 & $45,16 \%$ & $45,16 \%$ \\
S/ 5001 - S/ 10000 & 25 & $20,16 \%$ & $20,16 \%$ \\
S/ $10001-$ S/ 20000 & 13 & $10,48 \%$ & $10,48 \%$ \\
S/ 20 001- S/ 30000 & 6 & $4,84 \%$ & $4,84 \%$ \\
S/ $30001-$ S/ 60000 & 13 & $10,48 \%$ & $10,48 \%$ \\
S/ 60 001 - S/ 90000 & 4 & $3,23 \%$ & $3,23 \%$ \\
S/ 90 001 - S/ 120000 & 1 & $0,81 \%$ & $0,81 \%$ \\
TOTAL & 124 & $100.00 \%$ & $100.00 \%$ \\
\hline
\end{tabular}

Fuente: Elaboración propia

Tabla 2

Plazo aprobado

\begin{tabular}{lccc}
\hline Rango de plazo aprobado & Frecuencia & Porcentaje & Porcentaje Acumulado \\
\hline 1 a 6 meses & 14 & $11,29 \%$ & $11,29 \%$ \\
7 a 12 meses & 68 & $54,84 \%$ & $66,13 \%$ \\
13 a 18 meses & 24 & $19,35 \%$ & $85,48 \%$ \\
19 a 24 meses & 9 & $7,26 \%$ & $92,74 \%$ \\
25 a 30 meses & 3 & $2,42 \%$ & $95,16 \%$ \\
31 a 36 meses & 5 & $4,03 \%$ & $99,19 \%$ \\
37 a 42 meses & 0 & $0 \%$ & $99,19 \%$ \\
43 a 48 meses & 1 & $0,81 \%$ & $\mathbf{1 0 0 , 0 0 \%}$ \\
TOTAL & 124 & $100 \%$ & \\
\hline
\end{tabular}

Fuente: Elaboración propia

\section{DISCUSIÓN}

Este trabajo sostiene que la gestión crediticia incide en la morosidad tanto a nivel de evaluación del crédito como a las condiciones de este.

Las bases teóricas consideradas para el presente trabajo de investigación han permitido explicar, por un lado, el negocio bancario, sus riesgos y su regulación, y por otro lado, referenciar los productos, créditos y clientes que se tiene dentro de las evaluaciones de créditos; del mismo modo se señala la normatividad con respecto a las condiciones.

En cuanto a los estudios realizados sobre la incidencia de la gestión crediticia y la morosidad, la bibliografía señala que son varios los aspectos que inciden en la tasa de mora, desde lo que es una oferta excesiva de créditos, el otorgamiento de fondos a clientes con baja capacidad de pago, hasta el no contar con un plan para recuperar los créditos, entre otros. De la misma manera, el alto índice de morosidad se debe al manejo inadecuado de los protocolos de créditos, por lo que recomienda un nuevo diseño de políticas y estrategias para controlar y mejorar dichos procesos.

En otro punto, el crecimiento de la morosidad es una repercusión a raíz de una deficiente evaluación, que se atribuye a la falta de capacitaciones para los asesores, implicando factores que inciden en el incumplimiento de los pagos. El índice de morosidad afecta directamente a las distintas instituciones financieras, así como al desarrollo de las gestiones financieras de las mypes. Por lo que, siguiendo la línea de investigación, basado en el análisis de evaluación de microcréditos, esta es un apoyo que permitirá generar un conocimiento fáctico en cuanto a los factores importantes que inciden en el índice de morosidad. 
Lo cual no solo será en beneficio del área de microfinanzas de la agencia de Chorrillos del Banco Financiero del Perú, sino también que podrá trasladarse a otras área y entidades financieras, ya que la calidad de la cartera activa es determinante para las actividades y la rentabilidad, debido al impacto que tienen los costos asociados en la provisión derivada de la cartera de riesgo y las pérdidas asociadas a los créditos que no resultan liquidados por los deudores. Sin embargo, esta investigación señala que las condiciones de crédito inciden significativamente en la morosidad, específicamente, en cuanto a las condiciones del crédito, en el que se especifica que gran parte de los clientes con créditos vencidos no cuentan un aval, y de los pocos que cuentan con avales, parte de ellos no presentan ingresos. Las evaluaciones de crédito inciden significativamente en la morosidad, exponiendo que, el monto del crédito, el género del solicitante, la garantía otorgada y el estado civil resultaron determinantes en los niveles de mora. De igual modo, Tierra (2015), Ticse (2015) y Agurto y Córdova (2017) coinciden en que el primer factor que incide en el índice de morosidad es la deficiencia a nivel de la evaluación de los créditos, ya que genera un incremento desmedido de las colocaciones, contribuyendo a su vez al crecimiento del índice de morosidad, lo que concuerda con lo contrastado en la hipótesis específica 2, considerando dentro de ella el principal indicador de los números de casos que cumplen o no con la normativa establecida.

En base a las correlaciones obtenidas en la investigación se obtiene como conclusiones las siguientes:

- Las condiciones del crédito inciden significativamente en la morosidad del área de microfinanzas del Banco Pichincha del Perú.

- Las condiciones del crédito inciden significativamente en la morosidad del área de microfinanzas, siendo los aspectos relevantes que el tipo de productos predomina el activo fijo soles, representando un $66,94 \%$; asimismo, predominan los clientes nuevos con $42,74 \%$ y el rango de edad con mayor predominancia es de 34 a 46 años con $35,48 \%$.

- La evaluación crediticia incide significativamente en la morosidad del área de microfinanzas, encontrando que, en el caso de montos desembolsados impera el rango entre S/ 1001 a S/ 5000 soles, representando por el $45,16 \%$ de los créditos vencidos; asimismo, en cuanto a los plazos de créditos otorgados predomina los aprobados entre 7 a 12 meses representado por el $54,84 \%$ y los clientes con respaldo patrimonial solo representan $12,90 \%$.

\section{REFERENCIAS}

Aguilar, G., Camargo, G. y Morales, R. (2004). Análisis de la morosidad en el sistema bancario peruano Informe final de investigación. Instituto de Estudios Peruanos

Agurto, H., y Córdova, H. (2017). Determinantes de los niveles de morosidad en las cajas municipales de ahorro y crédito en el Perú - un estudio para el periodo 20012016 (Tesis de maestría). Universidad San Ignacio de Loyola, Lima, Perú. Recuperado de: http://repositorio. usil.edu.pe/bitstream/USIL/2719/1/2017_Agurto_Determinante-de-los-niveles-de-morosidad.pdf

Aranguiz, M., Gallegos , J., y Leal , A. (2018). Análisis de riesgo crediticio, propuesta del modelo credit scoring. Revista de la Facultad de Ciencias Económicas, 26(1), 181-207.

Economist Intelligence Unit. (2016). Microscopio Global 2016: Análisis del entorno para la inclusión financiera. The Economist. Recuperado de: http://graphics.eiu. com//assets/images/public/global-microscope-2016/ EIU_Microscope_2016_SP_web.pdf

Instituto Nacional de Estadística e Informática. (2019). Estructura empresarial, 2018. Recuperado de: https:// www.inei.gob.pe/media/MenuRecursivo/publicaciones_digitales/Est/Lib1703/libro.pdf

Martínez, M., Ríos, H., y Trejo, J. (2016). Análisis de la Administración del Riesgo Crediticio en México para Tarjetas de Crédito. Revista Mexicana de Economía y Finanzas, 11(1), 103 - 121.

Miño, K. (2014). Modelo de gestión financiera para la cooperativa de ahorro y crédito Pablo Muñoz Vega de la ciudad de Bolívar (Tesis de grado). Universidad Regional Autónoma de Los Andes, Quevedo, Ecuador. Recuperado de: http://dspace.uniandes.edu.ec/bitstream/123456789/1688/1/TUICYA008-2015.pdf

Pérez, E., y Tafur, R. (2015). Procedimientos de Evaluación a la Cartera de Clientes para Mejorar la Gestión Crediticia de "Repuestos y Accesorios Castillo S.R.L. (Tesis de pregrado). Universidad Privada Antenor Orrego, Trujillo, Perú. Recuperado de: http://repositorio.upao. edu.pe/bitstream/upaorep/1484/1/Perez_Quezada_ Proceedimientos_Cartera_Clientes.pdf

Ríos-Henckell, C. (2014). Perú: ¿Por qué es el campeón de las microfinanzas? Recuperado de: https://www.findevgateway.org/es/blog/2014/01/peru-por-que-es-elcampeon-de-las-microfinanzas 
Ticse, P. (2015). Administración del riesgo crediticio y su incidencia en la morosidad de Financiera Edyficar oficina Especial-El Tambo (Tesis de grado). Universidad Nacional del Centro del Perú, Huancayo, Perú. Recuperado de: http://181.65.200.104/bitstream/handle/UNCP/1620/TESIS\%20\%2810\%29.pdf?sequen$\mathrm{ce}=1$ \&isAllowed $=\mathrm{y}$

Tierra, J. (2015). El riesgo de crédito y la morosidad de La Cooperativa de Ahorro y Crédito Coorambato Cía. Ltda. del Cantón Ambato (Tesis de pregrado). Universidad Técnica de Ambato, Ambato, Ecuador. Recuperado de: https://repositorio.uta.edu.ec/bitstream/123456789/17398/1/T3252ig.pdf
Turpo, A. (2015). Análisis de la Calidad de cartera activa de la caja municipal de ahorro y crédito de Arequipa, agencia Macusani - periodo 2011 - 2013 (Tesis de maestría). Universidad Nacional del Altiplano, Puno, Perú. Recuperado de: http://repositorio.unap.edu.pe/ handle/UNAP/2202 